\title{
UNIQUENESS OF SOLUTIONS OF THE DIRICHLET AND NEUMANN PROBLEMS FOR HYPERBOLIC EQUATIONS
}

\author{
BY \\ EUTIQUIO C. YOUNG( $\left.{ }^{1}\right)$
}

\begin{abstract}
Conditions for uniqueness of solutions of the Dirichlet and Neumann problems are obtained for a singular hyperbolic equation involving a real parameter.
\end{abstract}

1. Introduction. It is well known that the Dirichlet problem for hyperbolic equations does not in general constitute a well-posed problem. In the case of the two-dimensional wave equation $u_{t t}-u_{x x}=0$, for instance, it is known that in order to determine the solution in a rectangle with sides having slopes \pm 1 , it is sufficient to prescribe its values on only two adjacent sides of the rectangle. On the other hand, Bourgin and Duffin [1] have shown that for rectangles with sides parallel to the coordinate axes, uniqueness of solution of the Dirichlet problem holds if and only if the ratio of the sides of the rectangle is an irrational number. Related investigations of the well-posedness of this problem have also been conducted by John [2] and Fox and Pucci [3]. More recently, the result in [1] has been extended by Dunninger and Zachmanoglou to the $n$-dimensional wave equation [4] and to more general hyperbolic equations in cylindrical domains [5]. A similar result on the Neumann problem has also been derived by Sigillito [6] for the $n$-dimensional wave equation. For singular equations of the fourth order, corresponding results have recently been obtained by Dunninger and Weinacht [7].

In this paper we present conditions for uniqueness of solutions of the Dirichlet and Neumann problems for the singular hyperbolic equation

$$
L u \equiv u_{t t}+(k / t) u_{t}-\left(a^{i j} u_{x_{i}}\right)_{x_{j}}+c u=0
$$

where the coefficients $a^{i j}$ and $c$ are functions of the variables $x_{1}, \ldots, x_{n}$ alone and $k$ is a real parameter, $-\infty<k<\infty$. Here the repeated indices are to be summed from 1 to $n$. The boundary value problems are considered in a cylinder $Q=D \times I$, where $D$ is a bounded domain in the $x=\left(x_{1}, \ldots, x_{n}\right)$ space and $I$ is the interval $0<t<T$. Necessary and sufficient conditions for uniqueness are given for different ranges of the parameter $k$.

Presented to the Society, January 21, 1971; received by the editors September 9, 1970 and, in revised form, January 8, 1971.

AMS 1969 subject classifications. Primary 3501, 3553, 3555, 3576.

Key words and phrases. Well-posed problem, uniqueness of solutions of the Dirichlet and Neumann problems for hyperbolic equations in cylindrical domains, eigenvalues and eigenfunctions.

(') The author was supported by NSF Grant GP-11543.

Copyright (C) 1971, American Mathematical Society 
We assume that the coefficient matrix $\left(a^{i j}\right)$ is symmetric and positive definite and that $c \geqq 0$ in $D$. Moreover, we assume that the functions $a^{i j}, c$, and the boundary $\partial D$ of $D$ are sufficiently smooth in order to allow the application of the divergence theorem and to ensure the existence of a complete set of eigenfunctions for the eigenvalue problems that arise in the sequel.

2. The Dirichlet problem. Consider the homogeneous Dirichlet problem

$$
L u=0 \text { in } Q, \quad u=0 \text { on } \partial Q .
$$

We shall prove uniqueness of solution by showing that every smooth solution of the problem vanishes identically in $Q$. In the case when $k>0$ however, we will see that every smooth solution vanishes identically in $\bar{Q}$ whenever it vanishes at $t=0$ and on the lateral surface of the cylinder $Q$.

THEOREM 1. Let $k>0$. If $u \in C^{2}(Q) \cap C^{1}(\bar{Q})$ is a solution of Lu=0 such that $u=0$ at $t=0$ and on $\partial D$ for $0 \leqq t<T$, then $u \equiv 0$ in $\bar{Q}$ for any value of $T$.

In order to prove this theorem we shall make use of the fact that every solution of equation (1) belonging to $C^{2}$ for $t>0$ and to $C^{1}$ for $t \geqq 0$ satisfies the condition $u_{t}(x, 0)=0$ for any nonzero $k$. In the special case when $\left(a^{i j}\right)$ is the identity matrix, this property was first established by Walter [8] for $c=0$ and then by Fox [9] for $c \neq 0$. The method used in [9], which was due to Zaremba and Asgeirsson and later improved by Walter, can be carried out here almost step for step to establish the same result for the more general equation (1) using the cylinder $Q$.

Proof of Theorem 1. We integrate the differential identity

$$
2 u_{t} L u=\left(u_{t}^{2}+a^{i j} u_{x_{i}} u_{x_{j}}+c u^{2}\right)_{t}-2\left(a^{i j} u_{x_{i}} u_{t}\right)_{x_{j}}+(2 k / t) u_{t}^{2}
$$

over the domain $Q_{s}=Q \cap\{0<t<s\}, s \leqq T$, and use the divergence theorem to obtain

$$
\int_{\partial Q_{s}}\left[\left(u_{t}^{2}+a^{i j} u_{x_{i}} u_{x_{j}}+c u^{2}\right) \nu_{t}-2 a^{i j} u_{x_{i}} u_{t} \nu_{j}\right] d S+2 k \iint_{Q_{s}} \frac{u_{t}^{2}}{t} d x d t=0
$$

where $\left(v_{1}, \ldots, v_{n}, v_{t}\right)$ denotes the outward normal vector on $\partial Q_{s}$. In view of the boundary conditions satisfied by $u$ and by the fact that $u_{t}(x, 0)=0$, equation (4) reduces to

$$
\left.\int_{D}\left(u_{t}^{2}+a^{i j} u_{x_{i}} u_{x_{j}}+c u^{2}\right)\right|_{t=s} d x+2 k \iint_{Q_{s}} \frac{u_{t}^{2}}{t} d x d t=0 .
$$

Since $\left(a^{i j}\right)$ is positive definite, $c \geqq 0$, and $k>0$, it follows that both terms in (5) must be zero. Thus

$$
\left.\int_{D}\left(u_{t}^{2}+a^{i j} u_{x_{i}} u_{x_{j}}+c u^{2}\right)\right|_{t=s} d x=0
$$

for $0 \leqq s \leqq T$. This implies that $u$ is a constant in $Q$. But $u=0$ at $t=0$, therefore $u \equiv 0$ in $\bar{Q}$ for any value of $T$. 
THEOREM 2. If $k \leqq 0$, then every solution $u \in C^{2}(Q) \cap C^{1}(\bar{Q})$ of the problem (2) vanishes identically in $Q$ if and only if

$$
J_{(1-k) / 2}\left(\lambda_{m}^{1 / 2} T\right) \neq 0
$$

where $\lambda_{m}(m=1,2, \ldots)$ are the nonzero eigenvalues of the problem

$$
\left(a^{i j} v_{x_{i}}\right)_{x_{j}}-c v+\lambda v=0 \text { in } D, \quad v=0 \text { in } \partial D,
$$

and $J_{p}(z)$ is the Bessel's function of the first kind of order $p$.

Proof. Suppose there exists a nonzero eigenvalue $\lambda_{p}$ of the problem (9) such that $J_{(1-k) / 2}\left(\lambda_{p}^{1 / 2} T\right)=0$. Let $v_{p}$ be the eigenfunction corresponding to $\lambda_{p}$. Then the function

$$
w(x, t)=t^{(1-k) / 2} J_{(1-k) / 2}\left(\lambda_{p}^{1 / 2} t\right) v_{p}(x)
$$

is a nontrivial solution of the problem (2) as is readily verified.

Conversely, if condition (8) holds, we integrate the differential identity

$$
w L u-u M w=\left(u_{t} w-u w_{t}+k u w / t\right)_{t}-\left[a^{i j}\left(u_{x_{i}} w-u w_{x_{i}}\right)\right]_{x_{j}}
$$

over the cylinder $Q_{s}^{T}$ enclosed by $Q$ between the planes $t=s$ and $t=T, 0<s<T$. The operator $M$ in (11) is the adjoint of $L$ and is given by

$$
M w=w_{t t}-k(w / t)_{t}-\left(a^{i j} w_{x_{i}}\right)_{x_{j}}+c w .
$$

By the divergence theorem we obtain

$$
\iint_{Q_{s}^{T}}(w L u-u M w) d x d t=\int_{\partial Q_{s}^{T}}\left[\left(u_{t} w-u w_{t}+k u w / t\right) v_{t}-a^{i j}\left(u_{x_{i}} w-u w_{x_{i}}\right) \nu_{j}\right] d S
$$

If $u$ is a solution of the problem (2) for $k \leqq 0$ and if we choose

$$
w(x, t)=t^{(1+k) / 2} J_{(1-k) / 2}\left(\lambda_{m}^{1 / 2} t\right) v_{m}(x)
$$

where $\lambda_{m}$ is a nonzero eigenvalue of the problem (9) and $v_{m}$ the corresponding eigenfunction, then $L u=0$ and

$$
M w=-t^{(1+k) / 2} J_{(1-k) / 2}\left(\lambda_{m}^{1 / 2} t\right)\left\{\left[a^{i j}\left(v_{m}\right)_{x_{i}}\right]_{x_{j}}-c v_{m}+\lambda_{m} v_{m}\right\}=0 .
$$

Thus the left-hand side of (13) vanishes and so we have

$$
\left.\int_{D}\left(u_{t} w-u w_{t}+k u w / t\right)\right|_{t=T} d x-\left.\int_{D}\left(u_{t} w-u w_{t}+k u w / t\right)\right|_{t=s} d x=0
$$

inasmuch as $w$ vanishes on $\partial D$ for $s \leqq t \leqq T$.

Now let $s$ approach zero. Since both $w_{t}$ and $w / t$ are bounded at $t=0$ and both $u$ and $u_{t}$ vanish there, the second term in (15) converges to zero. Hence in the limit (15) yields

$$
T^{(1+k) / 2} J_{(1-k) / 2}\left(\lambda_{m}^{1 / 2} T\right) \int_{D} u_{t}(x, T) v_{m}(x) d x=0
$$


In view of the condition (8), this implies that

$$
\int_{D} u_{t}(x, T) v_{m}(x) d x=0, \quad m=1,2, \ldots
$$

By the completeness of the set of eigenfunctions $\left\{v_{m}\right\}, m=1,2, \ldots$, it follows that $u_{t}(x, T)=0$.

Next, integrating the differential identity (3) over the cylinder $Q_{s}^{T}$ and using the fact $u(x, T)=u_{t}(x, T)=0$, we obtain

$$
\left.\int_{D}\left(u_{t}^{2}+a^{i j} u_{x_{i}} u_{x_{j}}+c u^{2}\right)\right|_{t=s} d x-2 k \iint_{Q_{s}^{T}} \frac{u_{t}^{2}}{t} d x d t=0 .
$$

Since $k \leqq 0$ it follows as in equation (5) that

$$
\left.\int_{D}\left(u_{t}^{2}+a^{i j} u_{x_{i}} u_{x_{j}}+c u^{2}\right)\right|_{t=s} d x=0
$$

for any $s, 0 \leqq s \leqq T$, which implies the conclusion of the theorem.

When $k=0$ condition (8) becomes $\sin \left(\lambda_{m}^{1 / 2} T\right) \neq 0$ which yields the result $\lambda_{m}^{1 / 2} T$ $\neq p \pi, p=1,2, \ldots$, previously obtained in [5].

Of special interest perhaps is the case when $\left(a^{i j}\right)$ is the identity matrix, $c=0$, and $D$ is the rectangle defined by $0<x_{i}<a_{i}, i=1,2, \ldots, n$. Equation (1) then reduces to the well-known Euler-Poisson-Darboux equation

$$
u_{t t}+(k / t) u_{t}-\Delta u=0 \quad\left(\Delta=\sum_{i=1}^{n} \partial^{2} / \partial x_{i}^{2}\right) .
$$

The corresponding eigenvalue problem (9) in this case is defined by

$$
\Delta v+\lambda v=0 \text { in } D, \quad v=0 \text { on } \partial D,
$$

for which the eigenvalues are given by

$$
\pi^{2} \sum_{i=1}^{n}\left(m_{i} / a_{i}\right)^{2}
$$

where $\left(m_{1}, \ldots, m_{n}\right)$ are $n$-tuples of positive integers. The condition ( 8$)$ then becomes

$$
J_{(1-k) / 2}\left\{\left[\sum_{i=1}^{n}\left(m_{i} / a_{i}\right)^{2}\right]^{1 / 2} \pi T\right\} \neq 0,
$$

$k \leqq 0$. In particular, when $k=0$ this gives

$$
T\left[\sum_{i=1}^{n}\left(m_{i} / a_{i}\right)^{2}\right]^{1 / 2} \neq m
$$

for all $(n+1)$-tuples $\left(m_{1}, \ldots, m_{n}, m\right)$ of positive integers, which is the result obtained in [4].

3. The Neumann problem. In the case of the homogeneous Neumann problem

$$
L u=0 \quad \text { in } Q, \quad \partial u / \partial n=0 \quad \text { on } \partial Q,
$$


where on the lateral surface of $Q$ the derivative $\partial u / \partial n$ is defined by

$$
\partial u / \partial n=a^{i j} u_{x_{i}} \nu_{j}
$$

$\left(\nu_{1}, \ldots, v_{n}\right)$ being the outward normal vector on $\partial D$, we have the following result.

THEOREM 3. If $u \in C^{2}(Q) \cap C^{1}(\bar{Q})$ is a solution of the problem (25), then $u \equiv 0$ (or $u=$ const if $c=0$ ) for $k \geqq 0$ if and only if

$$
J_{(1+k) / 2}\left(\lambda_{m}^{1 / 2} T\right) \neq 0
$$

where $\lambda_{m}(m=1,2, \ldots)$ are the nonzero eigenvalues of the problem

$$
\begin{aligned}
\left(a^{i j} v_{x_{i}}\right)_{x_{j}}-c v+\lambda v & =0 & & \text { in } D, \\
\partial v / \partial n & =0 & & \text { on } \partial D .
\end{aligned}
$$

Proof. The necessity of condition (26) actually holds for all $k$. For if there exists a nonzero eigenvalue $\lambda_{p}$ of (27) such that

$$
J_{(1+k) / 2}\left(\lambda_{p}^{1 / 2} T\right)=0
$$

then the function

$$
w(x, t)=t^{(1-k) / 2} J_{(k-1) / 2}\left(\lambda_{p}^{1 / 2} t\right) v_{p}(x),
$$

where $v_{p}$ is the eigenfunction corresponding to $\lambda_{p}$, constitutes a nontrivial solution of the problem (25) for any value of $k$. Indeed by (27) it follows that $L w=0$ and $\partial w / \partial n=0$ on $\partial D$ for $0 \leqq t \leqq T$. Moreover, since

$$
\partial w / \partial t=-\lambda_{p}^{1 / 2} t^{(1-k) / 2} J_{(1+k) / 2}\left(\lambda_{p}^{1 / 2} t\right) v_{p}(x)=O(t),
$$

it is clear that $w_{t}(x, 0)=0$ and by $(28) w_{t}(x, T)=0$. Thus the function (29) satisfies the boundary condition in (25) as well.

On the other hand, let $k \geqq 0$ and suppose that condition (26) holds. If $u$ is a solution of the problem (25) and if we choose

$$
w(x, t)=t^{(1+k) / 2} J_{(k-1) / 2}\left(\lambda_{m}^{1 / 2} t\right) v_{m}(x)
$$

then substitution of these functions in (13) leads again to equation (15). Since

$$
w_{t}(x, t)=\left[k t^{(k-1) / 2} J_{(k-1) / 2}\left(\lambda_{m}^{1 / 2} t\right)-\lambda_{m}^{1 / 2} t^{(1+k) / 2} J_{(k+1) / 2}\left(\lambda_{m}^{1 / 2} t\right)\right] v_{m}(x)
$$

it is clear that

$$
-w_{t}+k w / t=\lambda_{m}^{1 / 2} t^{(1+k) / 2} J_{(k+1) / 2}\left(\lambda_{m}^{1 / 2} t\right) v_{m}(x)=O\left(t^{1+k}\right) .
$$

Therefore, as $s$ is allowed to approach zero in (15), we obtain in the limit

$$
\lambda_{m}^{1 / 2} T^{(1+k) / 2} J_{(k+1) / 2}\left(\lambda_{m}^{1 / 2} T\right) \int_{D} u(x, T) v_{m}(x) d x=0
$$

which by (26) implies that

$$
\int_{D} u(x, T) v_{m}(x) d x=0, \quad m=1,2, \ldots
$$


By the completeness of the set of eigenfunctions $\left\{v_{m}\right\}$ of the problem (25), this implies that $u(x, T)=0$ if $c>0$ or $u(x, T)=$ const if $c=0$.

Let us assume that $c>0$. There remains to be shown that $u \equiv 0$ in $Q$. In this connection, the sufficiency proof of Theorem 2 (see equation (18)) does not permit us to make the desired conclusion since now $k \geqq 0$, except of course in the obvious case $k=0$. Thus for $k>0$ we need to use a different approach.

We integrate instead the differential identity

$$
\begin{aligned}
2 t^{\alpha} u_{t} L u= & {\left[t^{\alpha}\left(u_{t}^{2}+a^{i j} u_{x_{i}} u_{x_{j}}+c u^{2}\right)\right]_{t}-2 t^{\alpha}\left(a^{i j} u_{x_{i}} u_{t}\right)_{x_{j}} } \\
& +t^{\alpha-1}\left[(2 k-\alpha) u_{t}^{2}-\alpha a^{i j} u_{x_{i}} u_{x_{j}}-\alpha c u^{2}\right]
\end{aligned}
$$

for any real $\alpha>0$ over the cylinder $Q_{s}^{T}$ to obtain

$$
\begin{aligned}
-\int_{D} s^{\alpha}\left(u_{t}^{2}+a^{i j} u_{x_{i}} u_{x_{j}}+c u^{2}\right) & \left.\right|_{t=s} d x \\
& +\iint_{Q_{s}^{T}} t^{\alpha-1}\left[(2 k-\alpha) u_{t}^{2}-\alpha a^{i j} u_{x_{i}} u_{x_{j}}-\alpha c u^{2}\right] d x d t=0 .
\end{aligned}
$$

Letting $s$ approach zero in (34) and noting that the first term vanishes, we are then left with the convergent integral

$$
\iint_{Q} t^{\alpha-1}\left[(2 k-\alpha) u_{t}^{2}-\alpha a^{i j} u_{x_{i}} u_{x_{j}}-\alpha c u^{2}\right] d x d t=0
$$

for any real $\alpha>0$. If we rewrite this in the form

$$
(2 k-\alpha) \iint_{Q} t^{\alpha-1} u_{t}^{2} d x d t=\alpha \iint_{Q} t^{\alpha-1}\left(a^{i j} u_{x_{i}} u_{x_{j}}+c u^{2}\right) d x d t
$$

it becomes clear that (35) can hold if and only if the integral on each side of (36) vanishes. Thus in particular

$$
\iint_{Q} t^{\alpha-1}\left(a^{i j} u_{x_{i}} u_{x_{j}}+c u^{2}\right) d x d t=0
$$

from which the conclusion that $u \equiv 0$ in $Q$ follows.

If $c=0$ the discussion above implies that $u=$ const in $Q$. In the special case when $\left(a^{i j}\right)$ is the identity matrix, $c=0$, and $k=0$, condition (26) gives the result obtained in [6].

\section{REFERENCES}

1. D. G. Bourgin and R. Duffin, The Dirichlet problem for the vibrating string equation, Bull. Amer. Math. Soc. 45 (1939), 851-858. MR 1, 120.

2. F. John, The Dirichlet problem for a hyperbolic equation, Amer. J. Math. 63 (1941), 141-154. MR 2, 204.

3. D. W. Fox and C. Pucci, The Dirichlet problem for the wave equation, Ann. Mat. Pura Appl. (4) 46 (1958), 155-182. MR 21 \#3653. 
4. D. R. Dunninger and E. C. Zachmanoglou, The condition for uniqueness of solutions of the Dirichlet problem for the wave equation in coordinate rectangles, J. Math. Anal. Appl. 20 (1967), 17-21. MR 37 \#1807.

5. - The condition for uniqueness of the Dirichlet problem for hyperbolic equations in cylindrical domains, J. Math. Mech. 18 (1969), 763-766. MR 38 \#4817.

6. V. G. Sigillito, On the uniqueness of solutions of certain improperly posed problems, Proc. Amer. Math. Soc. 24 (1970), 828-831. MR 40 \#7612.

7. D. R. Dunninger and R. J. Weinacht, Improperly posed problems for singular equations of the fourth order, Applicable Analysis (to appear).

8. W. Walter, Über die Euler-Poisson-Darboux-Gleichung, Math. Z. 67 (1957), 361-376. MR 19, 1059.

9. D. W. Fox, The solution and Huygen's principle for a singular Cauchy problem, J. Math. Mech. 8 (1959), 197-219. MR 21 \#762.

Florida STATE University,

Tallahassee, Florida 32306 